\title{
iGreenhouse: A Case Study for Connecting Physical Devices into Mobile Social Networks
}

\author{
Jiajin Zhang ${ }^{1}$, Lichang Chen ${ }^{2}$,Xiaobo Cai ${ }^{1}$ and Quan Gao ${ }^{1}$ \\ ${ }^{1}$ School of Science and Information Engineering, Yunnan Agricultural University, \\ Kunming, China \\ ${ }^{2}$ Schools of Mechanic and Electronic Engineering, Yunnan Agricultural \\ University, Kunming, China, \\ *Corresponding Author: zjjclc@163.com
}

\begin{abstract}
Social networks have become extremely popular in recent years. A new requirement of connecting physical devices into social networks is emerging gradually. In this paper, we propose a case study to explore the effectiveness of connecting devices into mobile social networks for status monitoring and controlling. In our work, we developed iGreenhouse, a greenhouse can post its environment information automatically to the most popular mobile social networks Wechat in China, and Wechat users are able to remotely control the devices in the greenhouse easily. Experiment results show that our proposed approach is feasible, cost-effective and flexible. Therefore our solution can provide an alternative ubiquitous platform for monitoring and controlling of connected devices.
\end{abstract}

Keywords: Social Networks, Wireless Sensor and Actuator Networks, WeChat, Greenhouse

\section{Introduction}

Social Networks have become extremely popular and an important part of everyday life for interaction and communication in recent years [1].

Social Networks (e.g., Twitter, Facebook and linkedIn) make it possible for people to communicate and share information easily. And the Social Networks can be defined as web-based social spaces concentrate on facilitating communication, collaboration and content sharing among people who would like to publish, share and discuss short messages on the Web [2]. WeChat, a mobile chat application, can also be modeled as social networks, which launched by Tencent company in China in the early 2011. The user can send speech short letter, video, images and text through the rapidly network. WeChat supports for multiple people talking and chatting with good friends in more form which is similar to the SMS, Microblog, etc. The software of WeChat itself is complete free, and it can works on iOS, Android, Windows Phone, Symbian and Blackberry devices. By the end of 2013, Wechat achieved more than 300 million registered user accounts in only two years [3].

As a consequence, WeChat is the most popular mobile social networks in China. Additionally, WeChat enriches a software ecosystem by providing numerous Software Development Kits (SDK) making rich possibilities to application developers [4].

At present, a new requirement of connecting physical devices into social networks gradually is emerging. In this paper, we developed iGreenhouse combining a WeChat integrated wireless sensor/actuator networks (WSAN) and a greenhouse, which can post its environment information automatically to Wechat, At the same time, Wechat users are able to remotely control the devices in the greenhouse easily.

Our goal is to explore the effectiveness of connecting devices into social networks for status monitoring and controlling in the greenhouse, and to extend the social relationships 
between people to social relationships with their physical devices.

The rest of this paper is organized as follows. Section 2 presents an overview on related works. In Section 3, we introduce our proposed system architecture and implementation details and some experimental results are shown. Finally, Section 4 concludes the paper and presents the future work.

\section{Related Works}

There are some works that use social Networks as a platform for sensor network systems. There into, paper [5] provides a framework to globally share locally measured sensory readings based on Twitter. SoMoS [6] proposed a platformindependent middleware to manage Wireless Sensor Networks by integrating Social Network Services like Twitter, Facebook. Reference [7] describes that Twitter can provide an "open" publish-subscribe infrastructure for sensors and smartphones, and also pave the way for ubiquitous crowd-sourced sensing and collaboration applications.

Paper [8] has exploited the functionality and the Web 2.0 technologies provided by Facebook to transform the interaction with the Smart Home into a shared, social experience. Moreover, according to [9], a water dispenser can posts its working status automatically to Sina Weibo microblogging in China, and interacts with human beings in this social network. Twitter and Facebook are generally employed in the previous mentioned systems. However, it is a pity that these social networks can not be accessed in Chinese mainland. In addition, the above mentioned systems require extra effort for development and deployment.

Furthermore, approaches described above lack initiatives that provide remote control mechanisms oriented to open platforms. In view of the above-mentioned considerations, this paper presents a proposal that will address these issues. Our scheme leverages existing social networks as simple context architecture, and benefits from the existing functionalities and resources in the network.

\section{The Description of Proposed System}

\subsection{Architecture of Proposed System}

Our proposed system encompasses the simple three-layer architecture, which consists of: (1) the sensing/actuating layer in short-range and local networks, which is devoted to the data acquisition and digital control; (2) the network layer, which is aimed at transferring data and control commands across different networks; and (3) the application layer, where applications are deployed together with the middleware functionalities. Figure 1 shows the overall system structure of our proposed system.

In our work, we assume that workflow is as follows. (i) WSAN nodes are able to sample the environment parameters (such as temperature, humidity, soil moisture and light) in a greenhouse on demand or periodically, and (ii) the sensing data are conveyed to a gateway employed an Android smartphone via Bluetooth v4.0 connections. (iii) The gateway side application automatically processes the sensing data stored in a local database. And the gateway performs more complex tasks such as capturing an image of the greenhouse with a smartphone camera and wireless communicating over $\mathrm{WiFi}$ or cellular networks. (iv) The processed result includes the sensory data and the greenhouse image which can be further uploaded to a remote Wechat-based user application deployed at a cloud infrastructure server through WiFi or cellular networks (GPRS/3G/4G) and shared with social networks WeChat users. Then, (v) human users who can monitor these messages provide a full state of the plant growing, which enable to meet most of the requirement of environmental data collection and event detections. According to the richness of the 
data generated, human users can control their own WSAN nodes easily. Such as making a ventilator on or off. Here users only need a standard mobile Wechat client to send control commands and receive sensory information.

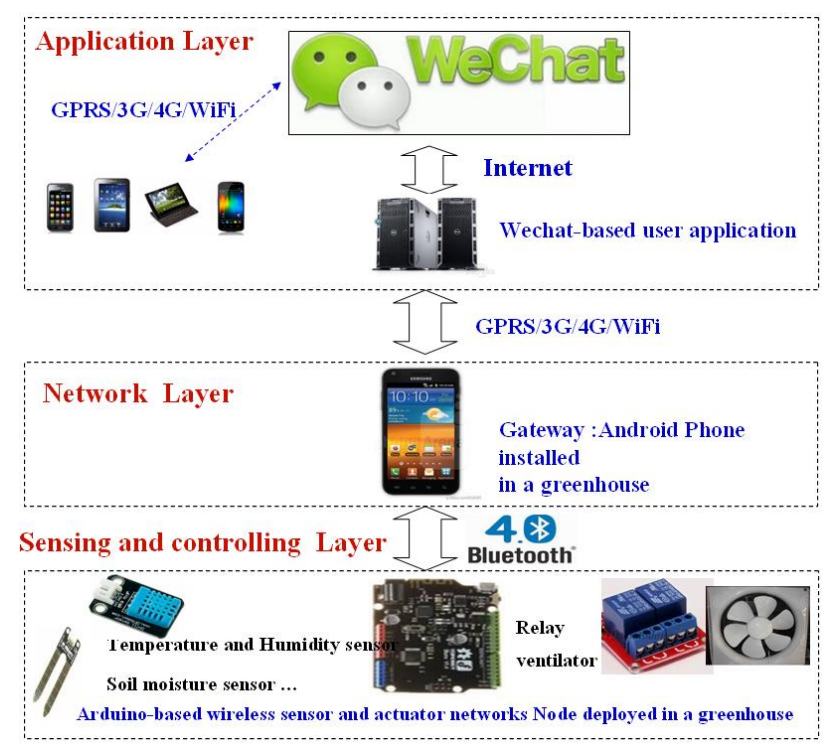

Figure 1. Architecture of our Proposed System

\subsection{Wireless Sensor/Actuator Node}

Rapid advances in embedded system technologies offer researchers a variety of new and inexpensive solutions for a wireless sensor networks (WSN) performs sensing, monitoring, and collecting information.

In addition, the concept of open source hardware, freely providing hardware designs, software programs and development efforts, is accepted widely. In our proposed system, with ease of use, low cost, low energy and standardized components and programming language cited as reasons for choosing Bluno Arduino serves as a WSAN node.

Bluno Arduino integrates with a TI CC2540 Bluetooth v4.0 chip with the Arduino UNO development board [10]. It allows wireless communication via Bluetooth Low Energy (BLE). And, for easy interfacing with the sensing and controlling units, including temperature-humidity sensor and light sensor, relay and stepper motor, etc., Bluno Arduino board was used, since Bluno Arduino can provide a rich library for interfacing with the above modules that quickly allows us build high-level WSN's applications.

Meanwhile, in our experiment system, A Bluno Arduino WSAN node (Figure 2) is mainly composed of a Bluno Arduino microcontroller board. One DHT11 is low cost digital temperature-humidity sensor, one soil moisture Sensor, one DFRobot BH1750 light intensity sensor (Figure 3), four relays and one ventilator, etc.

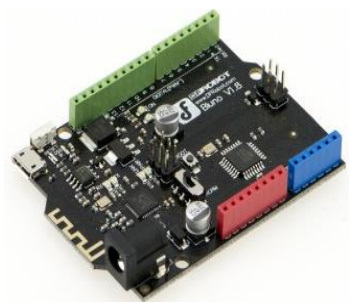

Figure 2. Bluno Arduino Board 


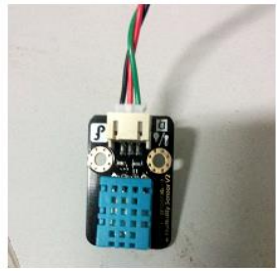

(a)

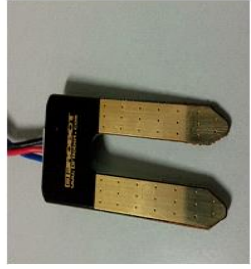

(b)

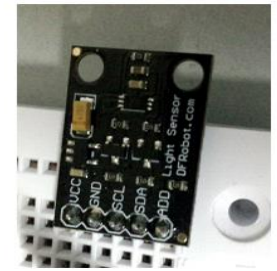

(c)

Figure 3. (A) Temperature-Humidity Sensor (B) Soil Moisture Sensor and (C) Light Intensity Sensor

\subsection{Gateway}

Compared with the traditional and dedicated gateways to bridge sensors and IP networks, Smartphone has significant advantages that make it a promising system platform, which include multiple short/long-distance network interfaces (e.g., WiFi, Bluetooth and cellular network), various integrated sensors, abundant computation and storage resources, and user-friendly interface and development environment.

Moreover, currently the price of Android smartphones has been dropping drastically. These features make it possible to build plenty of embedded sensing and controlling applications based on inexpensive off-the-shelf smartphones.

As a consequence, a powerful mobile computing platform could be used as an alternative gateway of WSN infrastructure, which is ubiquitous and scalable.

In our system, a M1-3 Android smartphone is deployed as a gateway, which runs Android OS 4.4 on $2.2 \mathrm{GHz}$ CPU, 2GB DDR2 memory, Bluetooth v4.0 BLE and a 13 megapixel camera with auto focus. This gateway not only hosts the Internet Proxy, but also adds an image monitoring functionality and periodically or on demand transmits images of key location of the greenhouse.

\subsection{Bluetooth v4.0 Wireless Sensor/Actuator Networks}

In this work, we adopt Bluetooth v4.0 for communications between a gateway Android smartphone and the WSAN nodes.

Bluetooth Low Energy (BLE) a feature of Bluetooth v4.0 under the standard IEEE.802.15.1 within a short range (up to 50 meters) can only support peer to peer and star typology, and as a result can not establish a meshed network. The lowenergy feature of a Bluetooth v4.0 can provide a more power-efficient solution for monitoring applications than existing solutions that use Bluetooth 2.1, ZigBee or WiFi [11]. Moreover, it has an advantage of being directly compatible with smartphones and tablets, without requiring a dongle or a gateway. This makes possible a new generation of WSN that interact directly with the user.

As seen in Figure 4, the sensor reading periodically sent by the Bluno Arduino WSAN nodes to a gateway through a Bluetooth v4.0 networks, is composed of a header, ID of WSAN node, values measured and checksum.

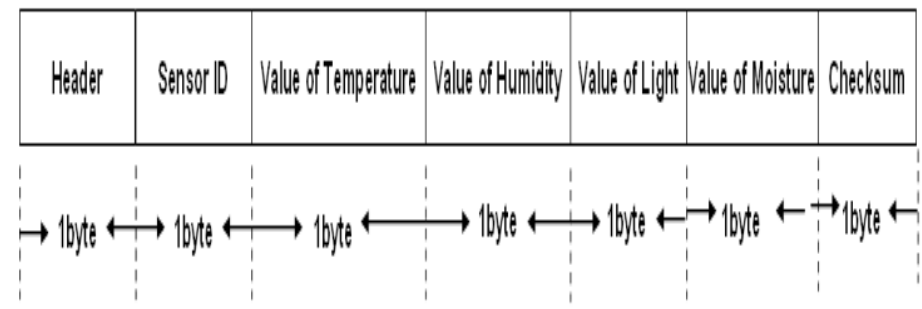

Figure 4. Format of Data Package 


\subsection{Gateway Side Application}

The WSAN nodes send their measurements to a gateway Android smartphone located at a greenhouse. Then the information is transmitted to WeChat and remote content management system via the gateway. As a result, a gateway side application is necessary. Figure 5 shows the relationship among components in our scheme.

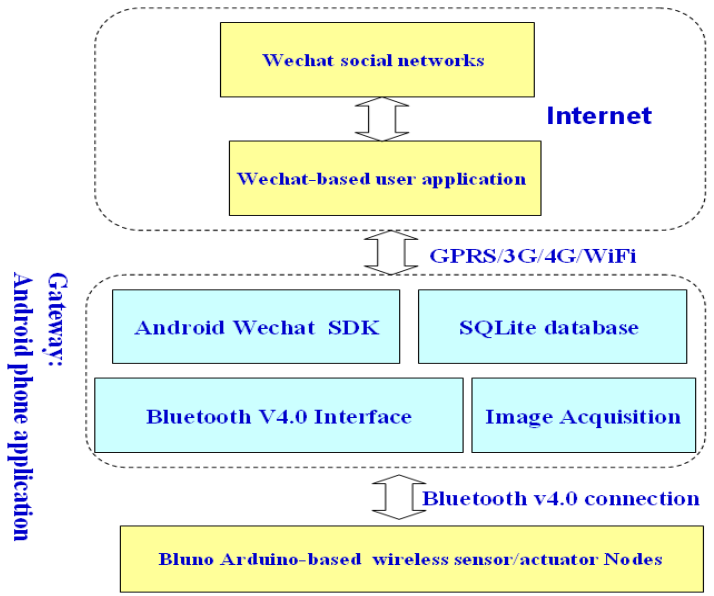

Figure 5. Block Diagram of Our Proposed System

As described above, the data from Bluetooth v4.0 WSAN nodes is transferred to the gateway side application. Then we can capture an image of the greenhouse with a camera of gateway smartphone through a camera Application Programming Interface (API) in our work. Next, all data including sensor data and image information is stored into a SQLite database and processed. After that, the application periodically uploads the processed text and image to the remote Wechatbased user application via Android WeChat SDK. Finally, message is conveyed to WeChat using PHP WeChat SDK. Android WeChat SDK, a key prerequisite for the realization of the gateway side application, consists of three core features including: 1) Log in/out, 2) Take and Renew an access token,and 3)Post / Delete text and image. To use the Android SDK to assist the development, developers have to register as a "developer" in WeChat developer open platform and obtain the corresponding App_ID and App_secret to complete the guided registration [4].

Authentication is need before Android SDK works. This SDK provides a WeChat API, when the program is initiated, the following key steps needs to be executed in a gateway side application.

(i) Register to Wechat

// "wx123456" is an APP_ID permitted by Wechat

// IWXAPI is a openapi interface provides the third party APP //interact with Wechat

private static final string APP_ID="wx123456"

private IWXAPI api;

private void regToWx () \{

// Through WXAPIFactory, archive a instantiation of IWXAPI api=WXAPIFactory.creatWXAPI(this,APP_ID,true);

// An APP_ID is registered into Wechat api.registerAPP(APP_ID); \} 
(ii) Send a request to Wechat

//An object of XTextObject is initiated

WXTextObject textObj = new WXTextObject();

textObj.text = text;

// An object of WXMediaMessage is initiated by WXTextObject

WXMediaMessage msg = new WXMediaMessage();

msg.mediaObject $=$ textObj;

//Send a text message

msg.description = text;

// Construct a Req

SendMessageToWX.Req req $=$ new SendMessageToWX.Req();

//The filed transaction is uniquely identified a request

req.transaction $=$ string. valueof $($ system.currenttimeMillis ()$)$;

req.message $=\mathrm{msg}$;

// Send data to Wechat using api

api.sendReq(req);

(iii) Receive a message from Wechat

//Add a WXEntryActivity classin the file Android Manifest

$<$ activity

android:name $="$.wxapi.WXEntryActivity"

android:lable=" @string/app_name"

android:exported="true"/>

After logging into WeChat, the gateway side application is allowed to get an access token by using App_secret. When the expiration of the access token occurs, this application renews the access token every five minutes.

The flow chart of the application is illustrated in figure 6.WSAN nodes sample the data every five minutes. After receiving all the data, the applic ation posts sensor data and image every five minutes.

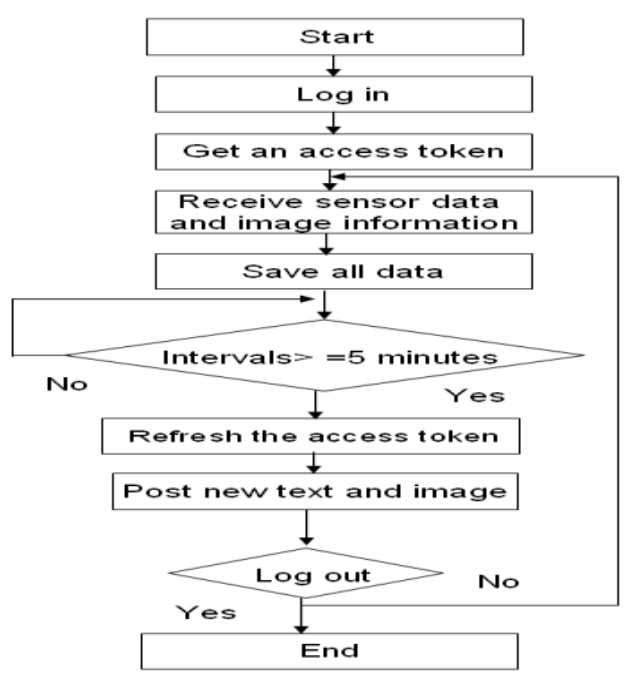

Figure 6. Flowchart of The Gateway Side Application

\subsection{Results of the Case Study}

To validate the concept of the proposed system, the developed system is tested by installing the environment condition sensing units and setting up a Bluetooth v4.0 
networks in a greenhouse located at Yunnan Agricultural University, which is deployed as shown in Figure 7.

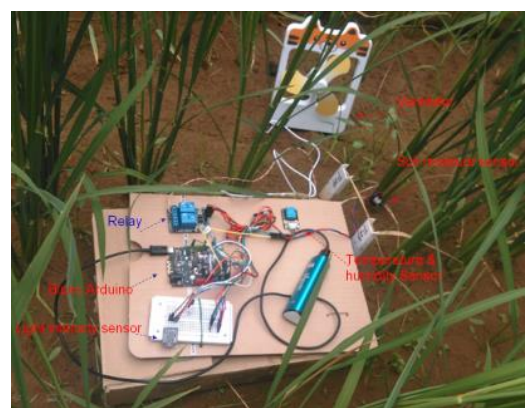

\section{Figure 7. Testbed of Bluno Arduino WSAN Node in Greenhouse}

Figure 8 illustrates practical results in a gateway Android smartphone. Here, the conditions in the greenhouse were measured at $22.5^{\circ} \mathrm{C}, 36 \%$ air relative humidity, $54 \%$ soil moisture and 163 Lux light intensity.

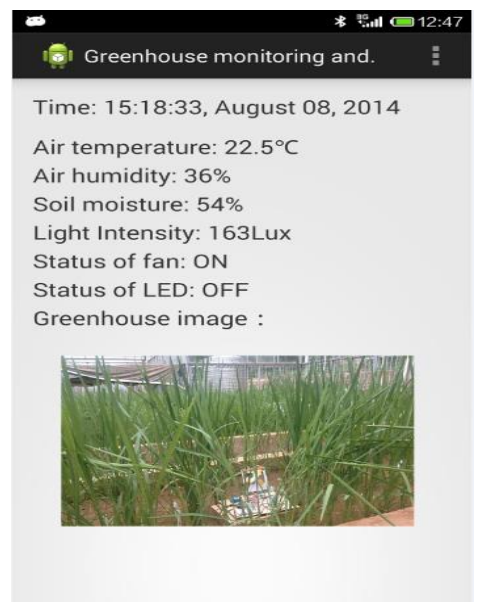

\section{Figure 8. Environment Information of a Greenhouse from a Gateway Android Smartphone}

Figure 9 depicts that posted message from a remote greenhouse are revealed on WeChat mobile client.

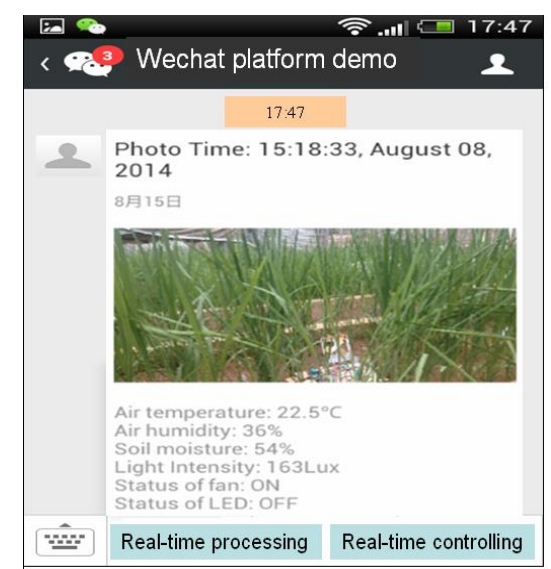

Figure 9. Posted Text and Image in Wechat Client of Android Smartphone 
Based on WeChat open platform, we developed an application deployed at a cloud infrastructure server using PHP program language, PHP Wechat interface SDK, Mysql database and Apache server. This application provides rich control functionalities mainly contain real time sensory data acquisition, image capture, auto/manual control option and ventilator on/off in the greenhouse.

Our Wechat-based user application menus are shown in Figure 10.

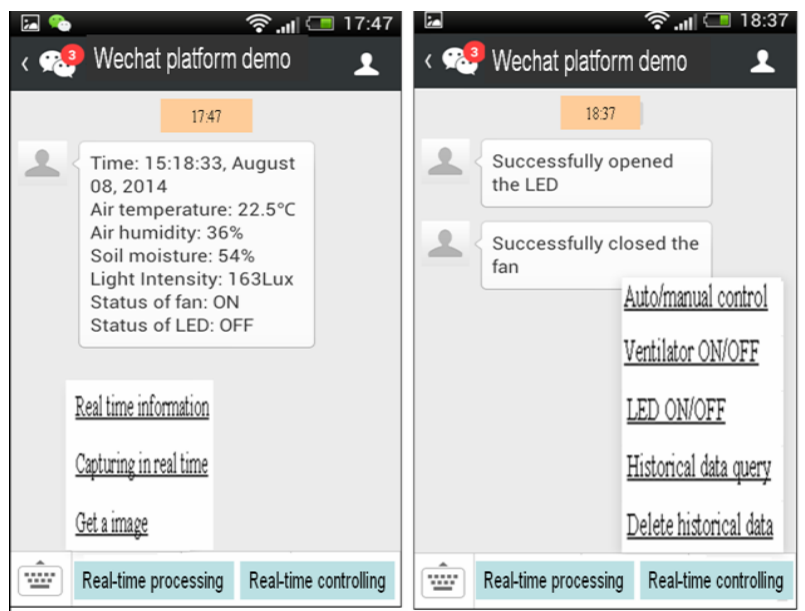

Figure 10. User Application Menus in Wechat Client of Android Smartphone

\subsection{Comparison between the Previous Solutions and Our Scheme}

Compared with the previous schemes mentioned earlier, there are some advantages in our proposed approach based on Wechat. The comparison of these systems is described as Table 1.

\section{Table 1. Comparison of Two Different Systems}

\begin{tabular}{|c|c|c|}
\hline Items & Our work & The previous solutions \\
\hline $\begin{array}{l}\text { Ease of software development and } \\
\text { hardware design }\end{array}$ & Very good & Medium \\
\hline Openness & Very good & Medium \\
\hline Remote control mechanism & Good & None \\
\hline Cost-benefit ratio & Very good & Medium \\
\hline
\end{tabular}

Besides, WeChat uses the OAuth2.0 authentication mechanism [12] for Wechat user authorization to complete the login process. Hence, this frees developer from writing OAuth application logic, and our method can reduce the overall system design complexity.

\section{Conclusions}

In this paper, utilizing WeChat social networks-based WSAN platform, we developed iGreenhouse as case study to verify the effectiveness of connecting physical devices into WeChat social networks for status monitoring and controlling in the greenhouse.

Moreover, Experimental result indicates our social networks based approach not only reduces the cost of development and deployment, but also benefits from existing interfaces and user behaviors in social networks. And our proposed solution can provide an alternative common environment for the interaction between human being and physical world. 
About the future work, we will intend to incorporate more advanced technology and develop a more reliable actuator networks to promptly react to changes of ambient parameters (e.g., $\mathrm{CH} 4, \mathrm{CO} 2$ ), and we may leverage the more usage of existing social networks in the precision agriculture.

\section{Acknowledgements}

This study was supported by an Academic Research Fund of Yunnan Agricultural University in 2014(YNAU14P1566).

\section{References}

[1] D. Zeng, S. Guo, and Z. Cheng, "The web of things, A survey", Journal of Communications, vol. 6. no. 6, (2011), pp. 424-438.

[2] D. M. Boyd and N. B. Ellison, "Social network sites", definition, history, and scholarship, ComputMediat Comm, vol. 13, no. 1, (2007), 210-230.

[3] Weixin .[Online]. Available: http://weixin.qq.com.

[4] Wechat open platform.[Online]. Available: https://open.weixin.qq.com.

[5] M. Baqer and A. Kamal, "S-sensors, Integrating physical world inputs with social networks using wireless sensor networks," in Intelligent Sensors, Sensor Networks and Information Processing (ISSNIP), 2009 5th International Conference on, (2009) December, pp. 213-218.

[6] B. He, M. Iwai, J. Chen, H. Chen and K. Sezaki, "SoMoS, an Interactive Social Network ServiceEnabled Middleware for Wireless Sensor Networks", In IEEE INSS2011, the 8th International Conference on Networked Sensing Systems, pp. 108-123.

[7] M. Demirbas, M. A. Bayir, C. G. Akcora, Y. S. Yilmaz and H. Ferhatosmanoglu, "A World of Wireless, Mobile and Multimedia Networks" Crowd-sourced Sensing and Collaboration using Twitter, Proceedings of the IEEE International Symposium on (WoWMoM), Montreal, (2010) June, Canada.

[8] A. Kamilaris and A. Pitsillides, "Social networking of the Smart Home", In IEEE 21st International Symposium on Personal Indoor and Mobile Radio Communications (PIMRC 2010), (2010) September.

[9] L. Chen, Y. Li, Z. Zheng, L. Zhang, D. He, X. Li, S. Zhao and S. Li, G. Pan, "WaterLady, A Case Study for Connecting Physical Devices into Social Networks", International Workshop on Green and Pervasive Software (GreenPS 2012), in conjunction with UIC 2012, Fukuoka, Japan, (2012) September 04-07.

[10] Arduino.[Online] .Available:http://arduino.cc.

[11] Bluetooth low energy.[Online] .Available: http://en.wikipedia.org/wiki/Bluetooth_low_energy.

[12] OAuth2.0.[Online].Available:http://oauth.net.

\section{Author}

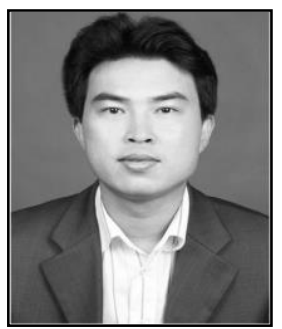

Jiajin Zhang, M.S. degrees in computer science ,Lecturer in School of Science and Information Engineering, Yunnan Agricultural University Scientific interest: the internet of things, embedded system, pervasive computing, cyber-physical system, Bioinformatics. 
International Journal of Smart Home

Vol. 9, No. 6 (2015) 